\title{
Quantitative Density Analysis of Ultra-Low Density Polymer Foams Using Various X-ray Imaging Techniques
}

\author{
Nikolaus L. Cordes ${ }^{1}$, Joseph Cowan ${ }^{1}$, Christopher E. Hamilton ${ }^{1}$, Kimberly A. Obrey ${ }^{1}$, and Brian M. Patterson ${ }^{1}$ \\ ${ }^{1 .}$ Los Alamos National Laboratory, Los Alamos, NM
}

Laboratory-based nano-scale X-ray computed tomography is a valuable tool for the visualization and quantification of microstructures within various materials. With approximately $150 \mathrm{~nm} 2 \mathrm{D}$ resolution, internal structures of a system can be probed non-destructively, which is not available with other imaging systems. Of significant interest is the microstructure of high internal phase emulsion (HIPE) chlorinated polystyrene foams for use in inertial confinement fusion targets.[1,2] In addition to local microstructure, another important property of polystyrene foams is the density of the foam; this property is traditionally measured using gravimetric methods. However, this is a bulk method which can be plagued with large uncertainties if the sample is of ultra-low density. Furthermore, this method yields no information of larger scale density gradients within the foam. A solution to this analytical problem is monochromatic X-ray radiographic imaging.[3] With this characterization technique, a quantitative density measurement is possible based on the X-ray transmission of the sample. In addition, density gradients within the polystyrene foams can be observed. Herein, we report the microstructural characterization of a series of HIPE polystyrene foams using laboratory-based nano-scale X-ray computed tomography. As can be seen in the nano-scale CT reconstructed slice, presented in Figure 1, these low-density foams contain an internal void microstructure. The densities of these foams were also measured from radiographs (Fig. 2) obtained non-destructively using a monochromatic X-ray imaging system utilizing a chromium source operating at $5.4 \mathrm{keV}$. The densities of these foams range from $\sim 70$ to $160 \mathrm{mg} \mathrm{cm}^{-3}$, as determined by X-ray imaging. The majority of the X-ray based density measurements agree well with measured gravimetric densities; however, a subset of the polystyrene foams exhibit relatively large diameters $(\sim 400 \mu \mathrm{m})$, resulting in low X-ray transmission $(\sim 0.9 \%)$ and large relative percent differences between the image-based measurements and gravimetric measurements (Fig. 3). This result highlights the need for a monochromatic X-ray imaging system which operates at higher energy which would allow for a relatively larger transmission through thick foam samples.

\section{References}

[1] Steckle, W. P., Jr.; Nobile, A., Jr., Fusion Science and Technology, 43 (2003), p.301

[2] Steckle, W. P., Jr.; Smith, M. E.; Sebring, R. J.; Nobile, A., Jr., Fusion Science and Technology, 45 (2004), p. 74

[3] Lanier, N.E; Hamilton, C.; Taccetti, J.M., Review of Scientific Instruments, 83 (2012), p. 10E521-1. 


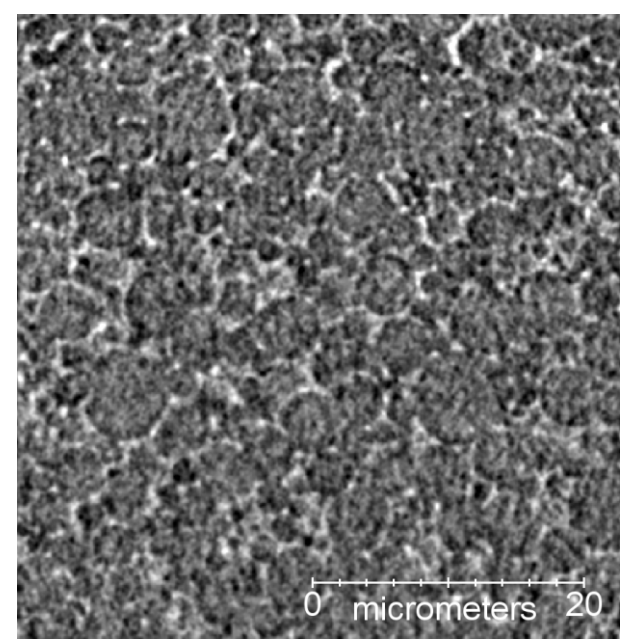

Figure 1. A reconstructed slice of polystyrene foam imaged using nano-CT in phase contrast mode. Bright areas correspond to foam ligaments, dark areas correspond to voids within the foam structure. Isotropic voxel size is $65 \mathrm{~nm}$.

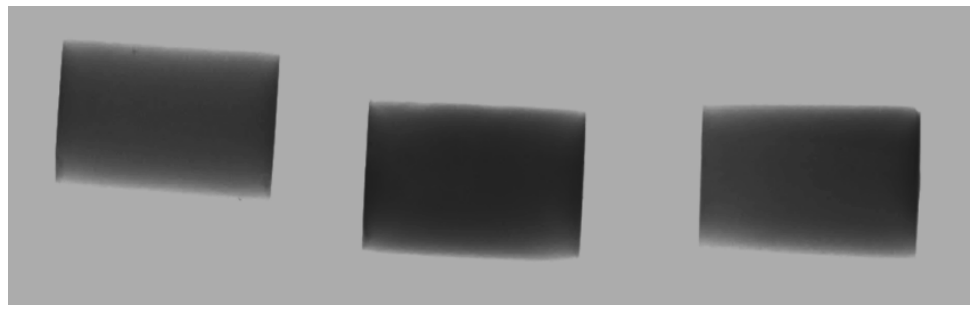

Figure 2. Radiographs of three polystyrene foams imaged using the monochromatic X-ray imaging system.

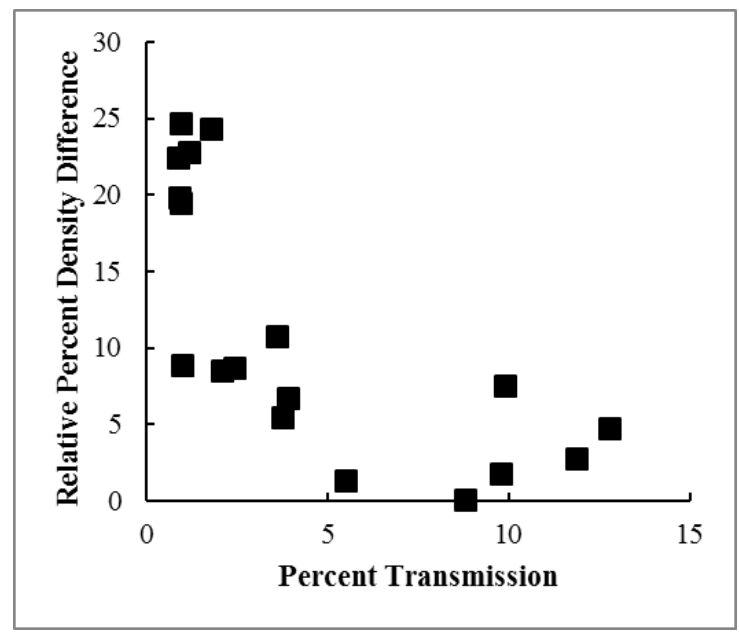

Figure 3. The relative percent density difference of the gravimetric density and the density determined from X-ray imaging as a function of percent X-ray transmission, highlighting the need for higher energy $\mathrm{X}$-ray source to adequately determine the density of thick polystyrene foams. 